\title{
CHRONOLOGIE DE L'HISTOIRE DU TIBET
}

\author{
Alice Travers
}

Outre-terre | Outre-Terre

2009/1 - n 21

pages 109 à 128

\section{ISSN 1636-3671}

Article disponible en ligne à l'adresse:

http://www.cairn.info/revue-outre-terre-2009-1-page-109.htm

Pour citer cet article :

Travers Alice, «Chronologie de l'histoire du Tibet »,

Outre-Terre, 2009/1 n²1, p. 109-128. DOI : 10.3917/oute.021.0109

Distribution électronique Cairn.info pour Outre-terre.

(c) Outre-terre. Tous droits réservés pour tous pays.

La reproduction ou représentation de cet article, notamment par photocopie, n'est autorisée que dans les limites des conditions générales d'utilisation du site ou, le cas échéant, des conditions générales de la licence souscrite par votre établissement. Toute autre reproduction ou représentation, en tout ou partie, sous quelque forme et de quelque manière que ce soit, est interdite sauf accord préalable et écrit de l'éditeur, en dehors des cas prévus par la législation en vigueur en France. II est précisé que son stockage dans une base de données est également interdit. 


\title{
Chronologie de l'histoire du Tibet ${ }^{1}$
}

\author{
Alice Travers $^{2}$
}

Les travaux archéologiques menés dans la seconde moitié du $\mathrm{xx}^{\mathrm{e}}$ siècle ont mis en évidence une présence humaine sur le haut plateau tibétain dès les périodes paléolithique et néolithique. La période dite « historique », c'est-à-dire pour laquelle on possède des sources écrites, remonte au début du VII ${ }^{\mathrm{e}}$ siècle, lors de la consolidation d'un puissant royaume au Tibet central. Cette chronologie, certes non exhaustive, mais basée sur les travaux d'historiens, retrace de la façon la plus factuelle possible les évolutions de l'histoire du Tibet, tant sur le plan intérieur que dans les relations protéiformes que celui-ci a nouées avec les grandes civilisations environnantes. Ces quatorze siècles d'histoire ont forgé une identité tibétaine dont l'écho a retenti avec une force particulière depuis mars dernier.

\section{Le grand empire tibétain de Pugyel (tib. Spu rgyal) en Asie centrale et la première diffusion du bouddhisme (c. 600-842)}

Entre le $\mathrm{VII}^{\mathrm{e}}$ et le $\mathrm{IX}^{\mathrm{e}}$ siècles, les " rois selon le Dharma » (tib. chos rgyal) mettent en place, grâce à une redoutable armée, un vaste empire centre-asiatique. Parmi les élites se développe une culture cosmopolite, bouddhique et polyglotte, dont la raison première est de pouvoir communiquer avec les pays vaincus ou li-

1. La chronologie proposée ici est principalement fondée sur les chronologies d'Anne-Marie Blondeau et Katia Buffetrille, cf. Anne-Marie Blondeau, Katia Buffetrille (éd.), Authenticating Tibet. Answers to China's 100 questions, Berkeley, University of California Press, p. 331-338 et de Dan Martin (THDL) qui s'est lui-même inspiré de la chronologie compilée par Michael Aris à partir de données de Christopher Beckwith, Hugh Richardson et David Snellgrove, de Helmut Hoffman et Melvyn Goldstein. D'autres ouvrages historiques d'intérêt général, dont le lecteur trouvera les références dans la bibliographie, ont permis de la compléter. Les noms tibétains sont donnés dans la translittération de Turrell V. Wylie. Je tiens à exprimer tous mes remerciements à ma directrice de thèse Heather Stoddard, ainsi qu'à Anne-Marie Blondeau, Fabienne Jagou et Françoise Robin pour leur relecture critique de ce travail et leurs suggestions.

2. Doctorante à l'université de Paris X-Nanterre et à l'Institut National des Langues et Civilisations Orientales (INALCO). 
mitrophes. L'écriture tibétaine est créée. En dépit de l'isolement géographique du plateau tibétain, une très grande ouverture aux civilisations voisines apparait.

Règne de Songtsen Gampo (tib. Srong brtsan sgam po) et début de la première diffusion du bouddhisme au Tibet

608 : Sous le règne de Namri Songtsen (tib. Gnam ri srong btsan), la première ambassade tibétaine est reçue à la cour de l'empereur chinois Yangdi (r. 604-617) de la dynastie des Sui (589-618).

617-649/650 : Règne de Songtsen Gampo (c. 604-649/650) et début de la période appelée « première diffusion du bouddhisme » (tib. snga dar) : conquête de nombreux royaumes indépendants dont le Shangshung (tib. Zhang zhung) à l'Ouest, expansion territoriale vers l'Est et le Nord-Est du haut plateau, invasion du Nord de l'actuel Yunnan et du Népal ; création d'un alphabet tibétain ; construction de plusieurs temples bouddhiques dont le Jokhang à Lhassa.

641 : Songtsen Gampo épouse la princesse chinoise Wencheng.

649-677 : Règne de Manglong Mangtsen (tib. Mang slong mang brtsan) : conquête du royaume Asha (tib. 'A zha), au Nord-Est du haut plateau tibétain ; première expansion tibétaine dans le Turkestan oriental, conquête d'oasis situées sur la route de la Soie.

680 : Incursions tibétaines nombreuses dans le Nord-Ouest du haut plateau et en Asie centrale.

692 : Perte temporaire du Turkestan oriental.

Règne de Trisong Detsen (tib. Khri srong Lde brtsan) et apogée de l'empire

755-797 : Règne de Trisong Detsen (742-797 (?)) : proclamation du bouddhisme religion d'État ; invitation de grands maîtres indiens comme Shantarakshita et Padmasambhava ; construction vers 779 du premier monastère bouddhique, Samyé (tib. Bsam yas), au Tibet central ; premières traductions de grands textes du bouddhisme indien en tibétain. Répression de l'ancienne religion tibétaine ; période d'expansion territoriale.

763 : Les Tibétains envahissent la capitale chinoise Chang'an, l'actuelle Xi'an.

786 : Occupation de l'oasis de Dunhuang jusqu'en 851.

790-c. 860 : Seconde expansion coloniale tibétaine dans le Turkestan oriental.

c. 792-794 : Controverse doctrinale à Samyé, appelée " Grand débat », au sujet de l'adoption du bouddhisme chinois ou indien. Le bouddhisme indien est choisi par l'empereur tibétain.

799-815 : Règne de Tridé Songtsen (tib. Khri lde srong brtsan) : influence grandissante du clergé bouddhique.

815-838 : Règne de Tri Ralpachen (tib. Khri ral pa can) : le traité de paix 
sino-tibétain de 822, dont témoigne le pilier érigé devant le temple du Jokhang à Lhassa, garantit la plupart des conquêtes tibétaines. Le Tibet comprend alors l'actuel Qinghai, une partie du Gansu et une partie de l'Ouest du Sichuan. Le traité définit les frontières et déclare que « les Tibétains seront heureux au Tibet et les Chinois heureux en Chine. »

\section{Dislocation de l'empire tibétain}

c. 842 : Assassinat du dernier empereur tibétain Langdarma (tib. Glang dar ma), accusé selon la tradition tibétaine d'avoir persécuté le bouddhisme car son influence grandissante affaiblissait l'empire.

851 : Perte de l'oasis de Dunhuang, située dans l'actuelle province du Gansu. Les Chinois chassent les Tibétains des oasis du Nord, les territoires du Turkestan ainsi que le Népal sont également perdus.

À la suite de querelles intestines et de nombreux revers militaires, parfois imputés à l'adoption du bouddhisme comme religion, l'empire tibétain est disloqué. Les survivants de la famille royale établissent des principautés au Tibet central et à la périphérie, dont le royaume de Ngari (tib. Mnga' ris) à l'Ouest du Tibet. Entre 852 et 970, après la chute de l'empire, le Tibet entre dans une période que l'on a pris l'habitude d'appeler le « siècle obscur », en raison du manque d'information et de sources le concernant. Les Tibétains l'appellent la « période de fragmentation » (tib. Bod sil bu'i dus skabs).

\section{Deuxième diffusion du bouddhisme au Tibet, instauration d'un puissant monachisme et des mécénats étrangers ( $\mathrm{X}^{\mathrm{e}}$ siècle-1642)}

À la fin du $\mathrm{x}^{\mathrm{e}}$ siècle, le Tibet est le théâtre d'un renouveau bouddhique venant des provinces périphériques du Tibet, de l'Est et de l'Ouest. Cette « deuxième diffusion du bouddhisme » (tib. phyi dar) est notamment le fait de nouvelles écoles bouddhiques (tib. gsar ma pa) qui se forment à partir du XI $\mathrm{I}^{\mathrm{e}}$ siècle, fondées par les disciples tibétains de maîtres indiens. Par contraste, à cette époque, les héritiers de la première diffusion du bouddhisme seront appelés Nyingmapa (tib. rnying ma pa ; litt. " les anciens »). Le pouvoir est partagé entre principautés rivales. Progressivement, les détenteurs laïcs du pouvoir temporel se soumettent aux monastères alors que dans d'autres cas, la même famille détient le trône abbatial et la charge politique. La relation de chöyön (tib. mchod yon, litt. « maître religieux-protecteur laïc »), qui permet dans un premier temps aux monastères d'être protégés par des princes locaux, est étendue dans un cadre nouveau, celui 
du mécénat avec des puissances étrangères, dans un premier temps mongoles.

\section{Renouveau bouddhique : retour aux sources indiennes et création de nouvelles écoles}

970 : Rinchen Zangpo (tib. Rin chen bzang po ; 958-1055) est envoyé au Cachemire par le roi Lhalama Yéshé Ö (tib. Lha bla ma ye shes 'od) du Ngari pour y étudier le métier de traducteur, inviter des maîtres et artistes indiens au Tibet et rapporter des textes. C'est le début de la deuxième diffusion du bouddhisme. S'initie de la sorte un mouvement de retour aux sources indiennes caractéristique de ce développement ultérieur du bouddhisme tibétain. Des Tibétains se rendent en Inde pour recevoir les enseignements de grands maitres.

978 : Lumé (tib. Klu mes) et Sumpa (tib. Sum pa) reviennent du Nord-Est au Tibet central, réintroduisant ainsi le monachisme bouddhique dans cette région.

1012-1096 : Vie de Marpa (tib. Mar pa) le Traducteur.

1040-1123 : Vie de Milarepa (tib. Mi la ras pa), le saint poète.

1042 : Le maître bengali Atisha (880-1054) arrive au Tibet. Ses enseignements sont à la source de l'école kadampa (tib. bka' gdams pa). Construction de monastères bouddhiques au Tibet.

1057 : Fondation du monastère de Réting (tib. Rwa sgrengs) par Dromtön (tib. 'Brom ston), disciple principal d'Atisha.

1073 : Fondation du monastère de Sakya (tib. Sa skya) par Könchog Gyeltsen (tib. Dkon mchog rgyal mtshan).

Fondation du monastère de Sangphu (tib. Gsang phu), première école de logique au Tibet.

1121 : Fondation du monastère de Dagla gampo (tib. Dwags lha sgam po), de la lignée kagyüpa (tib. bka' brgyud pa).

1158 : Fondation du monastère de Densatil (tib. Gdan sa mthil) qui deviendra le siège des Phagmodrupa (tib. Phag mo gru pa).

\section{Mécénat mongol et domination sakyapa}

1227 : Conquête et destruction du royaume Xixia par Gengis Khan.

1239 : Le général mongol Dorta le Noir envahit le Tibet sous les ordres de Godan Khan. Les princes mongols partagent le mécénat des lignées spirituelles au Tibet.

1247 : Sakya Pandita (1182-1251), hiérarque et érudit de l'école sakyapa, accepte l'invitation de Godan Khan, qui règne sur la région du Kokonor, et lui rend visite au Nord-Est du haut plateau tibétain, à Liangzhou.

1249 : Sakya Pandita reçoit de Godan Khan l'autorité spirituelle sur le Tibet. 
1260 : Phagpa (tib. 'Phags pa ; 1235-1280), neveu de Sakya Pandita, se rend sur ordre à la cour du Grand Khan Khubilaï (1215-1294), futur empereur de Chine et fondateur de la dynastie mongole des Yuan (1271-1368). Phagpa reçoit de Khubilaï le pouvoir de régence sur les treize myriarchies du Tibet (réorganisation territoriale et administrative du Tibet par les Mongols, comprenant le Tibet central ou U (tib. Dbus), le Tsang (tib. Gtsang), le Tibet de l'Ouest, le Kham (tib. Khams) et l'Amdo (tib. A mdo). Il est l'un des principaux artisans de la relation « maître religieux-protecteur laïc » entre religieux tibétains et dirigeants mongols.

1269 : Khubilaï demande à Phagpa de créer un alphabet mongol.

1290-1364 : Vie de Butön (tib. Bu ston), qui systématisa le canon bouddhique tibétain.

\section{Rivalités internes}

1358 : Changchub Gyeltsen (tib. Byang chub rgyal mtshan ; 1302-1364), héritier de la lignée des Phagmodrupa établie à Neudong (tib. Sne'u gdong) et rivale des Sakyapa au Tibet central, vainc les Sakyapa et prend le pouvoir. Il affranchit le Tibet de la domination mongole.

1357-1419 : Vie de Tsongkapa (tib. Tsong kha pa), fondateur de l'école qui sera connue plus tard sous le nom de Gelugpa (tib. Dge lugs pa ; litt. « les vertueux »). Construction du premier des trois monastères principaux ou « trois sièges » de l'école gelugpa, Ganden (tib. Dga' ldan) en 1409. Ses disciples fondent ensuite les monastères de Drepung (tib. 'Bras spungs) en 1416 et Sera (tib. Se ra) en 1419.

1368 : Fin de la dynastie mongole Yuan en Chine, début de la dynastie Han des Ming.

1414 : Fondation à Shigatse (tib. Gzhis ka rtse) du monastère de Tashilhünpo (tib. Bkra shis lhun po).

1426 : Visite du dernier pandit indien bouddhiste, Vanaratna. Le bouddhisme au Tibet n'empruntera désormais plus à l'Inde, où il a cédé la place à l'hindouisme et à l'islam.

1434 : Les Phagmodrupa commencent à perdre le pouvoir au profit des seigneurs de Rinpung (tib. Rin spungs), protecteurs d'une école religieuse rivale, celle des Karmapa (tib. Karma pa).

1498-1565 : Rivalités incessantes entre écoles gelugpa et karmapa et entre seigneurs locaux de Rinpung et du Tsang.

1578 : Sonam Gyatso (tib. Bsod nams rgya mtsho ; 1543-1588), troisième abbé du monastère gelugpa de Drepung, est invité par Altan Khan, chef des Mongols tümed converti au bouddhisme. Il reçoit le titre mongol de dalaï-lama (tib. Ta la'i bla ma; dalai signifie « océan » en mongol, traduction du tibétain gyatso). 
Il est lui-même le troisième dalaï-lama car ses deux incarnations précédentes, Gendun Drub (tib. Dge 'dun grub ; 1391-1474), neveu de Tsongkapa, puis Gendün Gyatso (tib. Dge 'dun rgya mtsho ; 1475-1542), premier et deuxième abbé de Drepung, sont reconnues rétroactivement comme premier et deuxième dalaï-lama. Début de la conversion des Mongols au bouddhisme gelugpa.

1588 : Mort de Sonam Gyatso. Sa réincarnation, le quatrième dalaï-lama Yönten Gyatso (tib. Yon tan rgya mtsho), est trouvée dans la parenté d'Altan Khan.

1612 : Le roi du Tsang, Karma Phüntsog Namgyel (tib. Karma Phun tshogs rnam rgyal) prend le pouvoir sur tout le Tibet central.

1617-1682 : Vie du cinquième dalaï-lama Ngawang Lobsang Gyatso (tib. Ngag dbang blo bzang rgya mtsho), appelé le « Grand cinquième ».

1622 : Intronisation du cinquième dalaï-lama au monastère de Drepung.

1623 : Karma Tenkyong (tib. Karma Bstan skyong), fils de Karma Phüntsog Namgyel, devient roi du Tsang.

\section{Tibet unifié des dalaï-lamas : gouvernement du Ganden phodrang (tib. Dga' ldan pho brang) (1642-1959)}

Grâce au soutien des Mongols, le cinquième dalaï-lama unifie, pour la première fois depuis la fragmentation de l'empire de Pugyel, un vaste territoire sous l'autorité d'un gouvernement ecclésiastico-nobiliaire, basé à Lhassa, le Ganden phodrang. En instaurant ce régime de type hiérocratique, il institutionnalise un système politique original, celui de l'union des pouvoirs temporel et du spirituel (tib. chos srid zung 'brel). Au XVIII' siècle, la dynastie mandchoue qui a pris le pouvoir en Chine en 1644 instaure un protectorat au Tibet, puis voit son pouvoir au Tibet s'estomper progressivement tout au long du XIX ${ }^{\mathrm{e}}$ siècle. À la fin du XIX et au début du $\mathrm{XX}^{\mathrm{e}}$ siècle, après une période de repli, le Tibet s'ouvre aux influences étrangère, jouissant d'une indépendance de fait. Le treizième dalaï-lama et une partie de l'élite politique tibétaine s'efforcent de mener une politique de réformes modernisatrices qui échoue dans un climat de luttes intestines et d'opposition des forces traditionalistes. À la fin du règne du treizième dalaï-lama et pendant les deux régences suivantes, le Tibet est dominé par les courants conservateurs. Certains Tibétains tentent de créer des partis politiques, inspirés des modèles républicains ou communistes, mais ils sont réprimés par le gouvernement tibétain. 


\section{Le " Grand cinquième " : unification du Tibet sous l'égide des Gelugpa alliés aux Mongols}

1642 : Gushri Khan, chef des Mongols qoshot, confère au cinquième dalaï-lama l'autorité spirituelle et temporelle sur le Tibet central, après avoir défait les princes du Tsang et des principautés du Kham. Le « Grand cinquième » crée le poste de régent (tib. sde srid), occupé par Sonam Rabten (r. 1642-1658) puis Sanggye Gyatso (tib. Sangs rgyas rgya mtsho ; r. 1653-1705). Le Khan qoshot devient protecteur du Tibet et Lhassa la capitale du Tibet des dalaï-lama. Construction du Potala, siège du gouvernement du Ganden phodrang, par le " Grand cinquième » et le régent Sanggye Gyatso.

1644 : Fin de la dynastie des Ming en Chine et début de la dynastie mandchoue des Qing.

1682 : Décès du cinquième dalaï-lama. Le régent Sanggye Gyatso garde la mort du dalaï-lama secrète jusqu'en 1696, afin de consolider le pouvoir du Ganden phodrang.

1683-1706 : Vie du sixième dalaï-lama, Tsangyang Gyatso (tib. Tshangs dbyangs rgya mtsho), homme de lettres fameux pour ses poèmes, mais peu intéressé par la vie monastique et le gouvernement.

1684 : Fin de la guerre avec le Ladakh qui accepte de payer tribut au Tibet.

1705 : Lhazang Khan, petit-fils de Gushri Khan et chef des Mongols qoshot, en accord avec l'empereur mandchou Kangxi (r. 1662-1723), attaque Lhasa, tue le régent et enlève le sixième dalaï-lama qui meurt en captivité.

1706 : Lhazang Khan tente d'imposer un dalaï-lama de son choix, Pékar Dzinpa (tib. Pad dkar 'dzin pa), mais les Tibétains, avec le soutien des Mongols dzungar, s'y opposent.

1717 : Les Mongols dzungar s'emparent de Lhassa et tuent Lhazang Khan.

\section{Protectorat mandchou (c. 1720-1911)}

1720 : L'empereur Kangxi chasse les Mongols dzungar du Tibet, installe le septième dalaï-lama Kesang Gyatso (tib. Bskal bzang rgya mtsho ; 1708-1757) qui n'exerce cependant pas de pouvoir temporel. Règne de Kangchenné (tib. Khang chen nas) et persécution de l'école nyingmapa.

1723 : Guerre civile à la suite du retrait des troupes mandchoues de Lhassa.

1725 : Une rébellion des princes mongols et tibétains de l'Amdo est matée par les Mandchous qui craignent la concurrence des Mongols en Asie centrale. L'Amdo est officiellement incorporé à l'empire mandchou.

1728 : Le noble tibétain Polané (tib. Pho la nas ; 1689-1747) prend le pouvoir avec l'appui des Mandchous. Deux représentants impériaux, les amban, de même qu'une garnison de 2000 soldats s'établissent à Lhassa. 
1740-1747 : Polané roi du Tibet. C'est le dernier dirigeant laïc que connaîtra le Tibet.

1750 : Le fils et successeur de Polané, Gyürmé Namgyel (tib. 'Gyur med rnam rgyal), est assassiné par les Mandchous après trois ans de règne. Les deux amban sont assassinés par les Tibétains lors d'émeutes. Une armée chinoise est envoyée pour les venger ; réorganisation du gouvernement par l'empereur mandchou.

1757 : À la mort du septième dalaï-lama, un régent ecclésiastique (tib. rgyal tshab) est nommé pour assurer l'interrègne jusqu'à la majorité du prochain dalaï-lama. Ce système sera utilisé jusque dans les années 1950.

1758-1804 : Vie du huitième dalaï-lama, Jampel Gyatso (tib. 'Jam dpal rgya mtsho), qui n'a jamais exercé le pouvoir.

1774-1775 : Mission de George Bogle, envoyé par le gouverneur de la Compagnie des Indes britanniques Warren Hastings auprès du troisième panchen-lama, deuxième lignée de réincarnation la plus importante au sein de l'école des Gelugpa et abbé du monastère de Tashilhünpo à Shigatsé.

1783 : Mission de Samuel Turner auprès du panchen-lama.

Le neuvième dalaï-lama Lungtog Gyatso (tib. Lung rtogs rgya mtsho ; 1806-1815), le dixième dalaï-lama Tsultrim Gyatso (tib. Tshul khrims rgya mtsho ; 1816-1837), le onzième dalaï-lama Kedrub Gyatso (tib. Mkhas grub rgya mtsho ; 1838-1855) et le douzième dalaï-lama Trinle Gyatso (tib. 'Phrin las rgya mtsho ; 1856-1875) meurent avant d'avoir pu exercer le pouvoir, qui est donc détenu par des régents successifs issus de monastères gelugpa et soutenus par les empereurs mandchous.

1788 : Première invasion des Gurkhas du Népal et défaite des Tibétains.

1790-1792 : Deuxième invasion gurkha, repoussée grâce à l'armée mandchoue. Début de la politique d'exclusion des étrangers au Tibet.

1792 : L'empereur mandchou Qianlong (r. 1736-1795) promulgue l' " Ordonnance en 29 articles » qui vise à réorganiser les institutions tibétaines, augmenter le pouvoir des amban et soumettre les affaires extérieures du Tibet à l'autorité de l'empereur. Dorénavant, les réincarnations des plus hauts dignitaires religieux (dont le dalaï-lama et le panchen-lama) doivent être choisies par un système de tirage au sort dans une urne d'or. Le système est cependant contourné par les Tibétains qui s'arrangent à ce que le nom de leur candidat soit tiré au sort.

1856-1875 : Conflit avec le Népal.

1865 : Les missionnaires chrétiens européens, qui s'étaient rendus nombreux au Tibet depuis le début du XVII ${ }^{\mathrm{e}}$ siècle, évacuent le pays et s'installent dans des régions périphériques. Pendant la deuxième moitié du XIX ${ }^{\mathrm{e}}$ siècle, l'accès au Tibet est interdit aux étrangers. Au début du Xx $x^{e}$ siècle, le pays s'ouvre sous la pression des intérêts commerciaux et politiques concurrents britanniques et russes.

1888 : Conflit sur la frontière du Sikkim entre Tibétains et Britanniques et première invasion britannique du Tibet. 
1890 : Les Britanniques annexent le Sikkim à leur empire des Indes. Convention sino-britannique complétée par les Régulations commerciales de 1893 autorisant les Britanniques à ouvrir une agence commerciale à Yatung (tib. Gro Mo), située à la frontière avec le Sikkim. Ces dispositions sont complétées par les Régulations commerciales de 1893. Les Tibétains refusent de reconnaître la validité de ces accords signés avec les Mandchous et sans eux.

1895 : Le treizième dalaï-lama Tubten Gyatso (tib. Thub bstan rgya mtsho ; 1876-1933) assume le pouvoir.

1896 : Tentative d'assassinat du treizième dalaï-lama par le régent Demo (tib. De mo).

1904 : Invasion britannique du Tibet sous les ordres de Francis Younghusband. Premier exil du treizième dalaï-lama vers la Mongolie puis vers la Chine en 1908. Défaite militaire des Tibétains qui signent un traité anglo-tibétain et acceptent de concéder des avantages commerciaux aux Britanniques, notamment la présence à l'intérieur de leurs frontières de trois agences commerciales à Yatung (tib. Gro mo), Gyantsé (tib. Rgyal rtse) et Gartok (tib. Sgar thog).

1907 : Après avoir occupé de larges parties du Kham oriental, le général mandchou Zhao Erfeng, surnommé le « boucher du Kham », les intègre dans une nouvelle province, le Xikang, et y mène une politique de sinisation et de militarisation.

1909 : Retour du treizième dalaï-lama à Lhassa. Peu de temps après, une avant-garde des troupes mandchoues de Zhao Erfeng occupe Lhassa.

1910 : Deuxième exil du treizième dalaï-lama, cette fois en Inde.

1911 : Révolution républicaine en Chine et chute de l'empire mandchou des Qing. La garnison mandchoue de Lhassa se rend, expulsion des Chinois présents au Tibet par les Tibétains.

\section{Indépendance de fait (1912-1951) et ouverture au monde moderne}

1912 : Retour du treizième dalaï-lama à Lhassa. Envoi de quatre enfants de l'aristocratie en Grande-Bretagne pour études à Rugby. Importation d'armes à partir de l'Inde.

1913 : Déclaration d'indépendance du Tibet par le treizième dalaï-lama ; signature du traité tibéto-mongol de reconnaissance mutuelle (la Mongolie a proclamé son indépendance dès 1911). Dans les années qui vont suivre, le Tibet se dote d'un drapeau, d'une monnaie et de timbres nationaux.

1914 : Convention tripartite de Simla entre la Grande-Bretagne, la Chine et le Tibet mais le traité n'est finalement pas ratifié par la Chine.

1918 : Accord de Rongbatsa (tib. Rong ba rtse) après la victoire des troupes 
tibétaines sur les troupes chinoises. Il délimite les frontières orientales du Tibet et de la Chine : le fleuve Yangtsé est reconnu comme frontière avec deux enclaves tibétaines sur la rive gauche; reconnaissance de fait de l'État tibétain.

1920-1921 : La mission de Charles Bell à Lhassa marque l'apogée des relations tibéto-britanniques. Aide britannique active pour l'équipement et la formation de l'armée tibétaine.

1923 : Fuite du neuvième panchen-lama (1883-1937) en Mongolie puis en Chine à la suite d'un différend entre son gouvernement local de Tashilhünpo et le gouvernement central de Lhassa. Ce dernier exigeait que Tashilhünpo paie des impôts dont il avait été exempté jusque-là afin de financer la nouvelle armée et différentes infrastructures. Ouverture d'une école anglaise à Gyantsé. Ligne de télégraphe Lhassa-Gyantsé achevée.

1924 : Construction d'une centrale hydro-électrique à Trapchi (tib. Grwa bzhi), près de Lhassa.

1925 : Réaction conservatrice. Le treizième dalaï-lama met un terme à la modernisation du pays, qu'il avait auparavant promue. Marginalisation de la faction progressiste anglophile.

1926 : Fermeture de l'école anglaise de Gyantsé.

1928 : Accession au pouvoir du gouvernement du Guomindang de Chiang Kai-shek. Instauration du gouvernement de Nankin (premier gouvernement centralisé depuis 1911 en Chine) et de la Commission des affaires tibétaines et mongoles.

1931 : Création par Künpela (tib. Kun 'phel lags), favori du dalaï-lama, du régiment d'élite Trongdra (tib. Grong drag dmag sgar).

1932 : «Testament» du treizième dalaï-lama qui exprime son inquiétude quant à l'avenir du bouddhisme au Tibet, en raison notamment de la destruction des monastères et du massacre des moines par les forces soviétiques en Mongolie.

1933 : Décès du treizième dalaï-lama.

1934 : Réting est nommé régent. Le gouvernement du Guomindang envoie une mission de condoléances dirigée par Huang Musong à Lhassa. Celui-ci laisse derrière lui un poste radio et une équipe qui ouvre un bureau de représentation « officieux » du gouvernement de Nankin.

Fondation par l'aristocrate tibétain Lungshar (tib. Lung shar) du Parti réformiste de l'Union heureuse qui veut réformer les institutions en donnant plus de pouvoir à l'Assemblée nationale. Un autre aristocrate, Changlochen (tib. Lcang lo can), est membre du parti.

Arrestation de Lungshar, accusé de complot contre le gouvernement et de tentative d'assassinat sur la personne du ministre Trimön (tib. Khri smon). Le gouvernement le condamne à être aveuglé. Exil de Künpela et Changlochen.

1935 : Naissance en Amdo du quatorzième dalaï-lama Tenzin Gyatso (tib. 
Bstan 'dzin rgya mtsho). Deux ans plus tard, une mission le reconnaît grâce aux indications de Réting et du neuvième panchen-lama.

1936 : Les Britanniques installent une mission permanente à Lhassa.

1939 : Fondation au Sichuan du Parti communiste tibétain par des étudiants originaires du Kham annexé, sous l'égide de Bapa Puntsog Wangyel (tib. 'Ba' pa Phun tshogs dbang rgyal). Ce parti nationaliste vise à mettre en place un Tibet indépendant et révolutionnaire. Bapa Puntsog Wangyel est banni du Tibet en 1949.

Fondation à Kalimpong (Inde) d'un parti inspiré de l'idéal républicain chinois, le Parti progressiste tibétain, par Künpela, Changlochen et le moine Gendün Chompel (tib. Dge 'dun chos 'phel ; 1905-1951).

1940 : Intronisation du quatorzième dalaï-lama.

1941 : Fin de la régence de Réting : le quatorzième dalaï-lama atteint l'âge de prononcer ses vœux monastiques traditionnellement reçus par le panchen-lama ou le régent. Le panchen-lama est trop jeune et il est de notoriété publique que le régent Réting ne respecte pas les vœux de célibat ; ce dernier se voit donc contraint de démissionner en faveur du moine conservateur Taktra (tib. Stag sgra ; 1874-1952).

1942 : Un ministère des Affaires étrangères est instauré (après une première tentative en 1911 consécutivement au retour du treizième dalaï-lama de son exil indien).

1943 : Mission américaine de Tolstoy et Dolan à Lhassa pour explorer les possibilités de convoiement de renforts américains à l'allié Chiang Kai-shek par le Tibet; refus du gouvernement tibétain.

1944-1945 : Tentative avortée d'ouvrir une école anglaise à Lhassa.

1946 : Le Parti progressiste tibétain est démantelé à Kalimpong. Emprisonnement de Gendün Chompel à Lhasa.

Une mission tibétaine de félicitations aux Alliés pour leur victoire se rend en Inde. Délagation tibétaine à la conférence panasiatique qui se tient à Delhi.

1947 : Échec du complot de Réting contre Taktra et révolte du collège monastique Sera che (tib. Se ra byes) qui le soutenait. Le gouvernement tibétain réprime la révolte, emprisonne Réting qui décède peu après en prison et épure son administration des partisans de ce dernier.

Indépendance de l'Inde, fin des intérêts britanniques au Tibet, la mission britannique devient mission indienne.

1948 : Une mission commerciale tibétaine se rend en Chine, aux États-Unis et au Royaume-Uni.

1949 : Chute du Guomindang, fermeture de la mission chinoise à Lhassa et expulsion de tous les Chinois du Tibet. Instauration de la République populaire de Chine (RPC). Mao Zedong appelle à la « libération pacifique » du Tibet.

1950, octobre : L'invasion du Tibet commence : l'Armée populaire de libé- 
ration attaque la ville de Chamdo (tib. Chab mdo) dans les territoires contrôlés par le gouvernement tibétain. L'armée tibétaine est rapidement vaincue. À la demande de l'Assemblée nationale tibétaine, le quatorzième dalaï-lama, âgé de 15 ans, assume le pouvoir. Le gouvernement tibétain lance un appel à l'ONU. Fuite du dalaï-lama à Yatung.

\section{Le Tibet chinois depuis 1951}

Au début des années 1950, alors que l'incorporation du Tibet à la RPC semble s'opérer, progressivement, au Tibet central, les agissements radicaux des communistes en Amdo et au Kham oriental mettent en lumière une volonté d'imposer leur politique tant sur le Tibet central que sur les provinces orientales indépendamment de la culture et de l'environnement naturel et social qui est le leur. La répression du soulèvement de Lhassa, en mars 1959, et la fuite du dalaï-lama en Inde marquent la fin du gouvernement traditionnel tibétain. Dès avant la Révolution culturelle et jusqu'au début des années 1980, la culture tibétaine subit une destruction massive. La politique de libéralisation et d'ouverture lancée par Deng Xiaoping en 1978 permet un renouveau culturel et religieux relatif au Tibet. Les manifestations qui ont lieu à Lhassa entre 1987 et 1989 traduisent cependant la persistance d'un fort sentiment identitaire. Le gouvernement chinois compte sur le développement économique en Région autonome du Tibet pour éradiquer ces revendications. Mais les manifestations du printemps 2008, dans toutes les régions de culture tibétaine de la RPC, semblent remettre en cause l'efficacité de cette politique.

Incorporation du Tibet à la République populaire de Chine et politique " gradualiste » de réformes menée par Mao Zedong au Tibet central

1951 : Signature à Pékin le 23 mai de l'accord en dix-sept points par lequel les représentants du gouvernement tibétain acceptent que le Tibet fait partie de la Chine en échange de l'autonomie interne. Ils reçoivent la garantie qu'il n'y aura aucune ingérence dans leur système traditionnel politique et religieux et que le statut du dalaï-lama sera respecté. Le dalaï-lama et son gouvernement sont informés de la signature de l'accord le 26 mai. Retour du dalaï-lama à Lhassa.

1954 : Le dalaï-lama et le panchen-lama se rendent à Pékin et y rencontrent Mao Zedong.

1955 : Dissolution de la province du Xikang et incorporation de tout le Kham oriental aux provinces du Sichuan et du Yunnan. L'Amdo correspondant globalement à la majeure partie de la province du Qinghai et à des zones dans les provin- 
ces du Gansu et du Sichuan. Soulèvements à la suite de la collectivisation forcée, bombardement des monastères au Kham. L'afflux de réfugiés du Kham oriental et de l'Amdo vers Lhassa commence. Création de l'Armée nationale volontaire de défense, une organisation khampa qui lutte contre les Chinois.

1956 : Le gouvernement chinois met en place le Comité préparatoire de la Région autonome du Tibet (CPRAT) pour remplacer le gouvernement tibétain. Le dalaï-lama et le panchen-lama se rendent en Inde pour les célébrations de la Buddha Jayanti (2 500 anniversaire de la naissance de Bouddha).

1957 : Création dans la région du Lhokha (tib. Lho kha) du mouvement de résistance Chushi gangdrug (tib. Chu bzhi sgang drug; litt. "Quatre rivières, six montagnes ») par des guerriers khampa. Début de l'aide de la CIA aux résistants.

1958 : Lancement du « Grand Bond en avant » en RPC. Rébellion massive de l'Amdo, fortement réprimée.

1959, 10 mars : Soulèvement à Lhassa et répression. Fuite du quatorzième dalaï-lama en Inde, suivi par environ 80000 Tibétains.

Le premier ministre Zhou Enlai annonce l'abolition de l'ancien gouvernement tibétain.

Le dalaï-lama fonde en exil un gouvernement qu'il installe à Mussorie, plus tard en 1960 à Dharamsala dans le Nord de l'Inde.

Le dalaï-lama dénonce l'accord en dix-sept points comme arraché sous la contrainte à ses représentants.

Le panchen-lama est nommé président du CPRAT.

L'ONU adopte une première résolution sur le Tibet appelant au « respect des droits fondamentaux du peuple tibétain et de sa spécificité culturelle et religieuse $»$.

\section{Radicalisation des réformes et Révolution culturelle}

1960 : Une base de guérilla tibétaine s'installe au Mustang (Népal) pour y organiser la résistance armée aux forces chinoises. Le roi du Népal qui signe des accords frontaliers avec la Chine va bientôt s'opposer à ces activités de résistance.

1960-1963 : Construction de la route Lhassa-Kathmandu.

1961 : L'ONU adopte une deuxième résolution reconnaissant le droit à l'autodétermination du peuple tibétain.

1962 : Guerre entre la Chine et l'Inde à propos de la frontière sino-indienne, la « ligne Mac-Mahon » décidée lors de la conférence de Simla en 1914 mais non reconnue par la Chine. La Chine inflige à l'Inde une lourde défaite. Malgré une nette amélioration des relations entre les deux pays, la question des frontières n'est toujours pas réglée en 2008. 
1963 : Le dalaï-lama promulgue en exil une constitution démocratique, fondée sur la Déclaration universelle des Droits de l'homme.

1964 : Arrestation à Lhassa du dixième panchen-lama en raison de son soutien au dalaï-lama (il sera réhabilité en 1978).

1965 : Création de la Région autonome du Tibet (RAT), qui n'englobe que les Tibet central et occidental, et le Kham occidental.

1965 : L'ONU adopte une troisième résolution sur le Tibet renouvelant son « appel à la cessation des toutes les pratiques qui privent le peuple tibétain des Droits de l'homme et des libertés fondamentales. »

1966 : Lancement de la Révolution culturelle, destruction massive des édifices religieux (monastères, temples, stupas, bibliothèques et images sacrées) et civils (palais, manoirs, forteresses). Interdiction de la vie monastique, rééducation et emprisonnement d'un grand nombre de Tibétains laïcs et religieux.

1970 : Création du Tibetan Youth Congress, la plus grande organisation politique non gouvernementale en exil, basée à Dharamsala.

1971-1972 : Les visites en Chine de Kissinger et de Nixon marquent le rapprochement sino-américain et la fin de l'aide de la CIA aux résistants tibétains.

1976 : Mort de Mao Zedong et fin de la Révolution culturelle.

1978 : Deng Xiaoping annonce une politique de libéralisation. Début de la reconstruction de monastères. Libération d'un grand nombre d'anciens fonctionnaires du gouvernement traditionnel tibétain qui avaient été emprisonné dès 1959 ou lors de la Révolution culturelle. Le panchen-lama est libéré et nommé membre du Comité consultatif politique du peuple chinois, il se fera l'avocat de la cause tibétaine jusqu'à sa mort.

1979 : Visite de la première délégation du gouvernement tibétain en exil au Tibet.

Renouveau culturel et religieux et réaffirmation du sentiment identitaire tibétain

1980 : Visite du secrétaire du Parti communiste chinois Hu Yaobang au Tibet, réformes politiques et économiques, libéralisation religieuse relative.

Visites des deuxième et troisième délégations du gouvernement tibétain en exil au Tibet.

1981 : Le secrétaire général du Parti communiste chinois Hu Yaobang annonce une « Politique en cinq points envers le dalaï-lama » dans laquelle il demande au dalaï-lama et à son entourage de revenir en RPC, promet que le dalaï-lama jouira du même statut politique et des mêmes conditions de vie qu'avant 1959 à condition qu'il ne réside pas en RAT.

1982 : Une délégation du gouvernement tibétain en exil se rend à Pékin pour traiter avec le gouvernement chinois mais aucun accord n'est conclu. 
Promulgation de la Constitution chinoise qui garantit la liberté de croyance religieuse pour tous les citoyens n'appartenant pas au Parti communiste chinois.

1984 : Selon les estimations du gouvernement tibétain en exil, la présence chinoise au Tibet aurait directement causé la mort de plus d'un million de Tibétains. Le chiffre n'est pas vérifiable.

1987 : Le dalaï-lama propose au Congrès américain son plan de paix en cinq points afin de résoudre la question du Tibet par des négociations avec le gouvernement chinois.

Émeutes à Lhassa et répression.

1988 : Le dalaï-lama présente au Parlement européen sa « Proposition de Strasbourg » : les trois provinces tibétaines - Tibet central, Kham et Amdo - seraient réunies en un « Grand Tibet »; en échange de quoi il accepterait l'autonomie au sein de la RPC, renonçant à l'indépendance et concédant au gouvernement chinois la responsabilité de la défense et des relations extérieures. Cette prise de position aliène à son approche pacifiste les Tibétains indépendantistes tandis que la première condition demeure irrecevable pour le gouvernement chinois.

Émeutes à Lhassa et répression.

1989 : Le prix Nobel de la paix est décerné au dalaï-lama. Nouvelles émeutes à Lhassa et répression. Instauration de la loi martiale (levée en avril 1990) dans toute la RAT par Hu Jintao, alors secrétaire de la RAT.

Mort du dixième panchen-lama.

Les manifestations des partisans de la démocratisation sur la place Tian'anmen à Pékin sont violemment réprimées.

1991 : La sous-commission de l'ONU sur la prévention de la discrimination et la protection des minorités adopte une résolution sur " La situation du Tibet », exprimant son « inquiétude due aux rapports permanents concernant la violation des Droits de l'homme et des libertés, qui menace l'identité spécifique culturelle, religieuse et nationale du peuple tibétain ». En octobre, le président des États-Unis, George Bush, ratifie une résolution du Congrès déclarant que le Tibet «pays occupé ».

1992 : Le dalaï-lama publie les « Lignes directrices pour la politique du Tibet futur et les traits principaux de sa Constitution » annonçant que, dans un futur Tibet libre, il renoncerait à son pouvoir temporel en faveur d'un gouvernement élu démocratiquement et que le gouvernement en exil serait dissous.

Arrivée au pouvoir au Tibet de Chen Kuiyuan (nommé secrétaire adjoint puis secrétaire du Comité du Parti communiste chinois pour la RAT) dont la politique tend à l'assimilation culturelle du Tibet à la Chine.

1993 : Rupture par le gouvernement chinois des relations officielles avec Dharamsala.

1995 : Le dalaï-lama reconnaît comme onzième panchen-lama l'enfant Gendun Chökyi Nyima (tib. Dge 'dun Chos kyi Nyi ma) aussitôt enlevé par les 
autorités chinoises. Le gouvernement chinois le remplace par un autre enfant de son choix, Gyeltsen Norbu (tib. Rgyal mtshan Nor bu), après utilisation du système ancien du tirage au sort dans l'urne d'or. Les relations entre Pékin et Dharamsala se détériorent.

1996 : Campagne de grande ampleur de rééducation patriotique et de dénonciation du dalaï-lama en RAT. Émeutes à Lhassa. La campagne politique s'étend à l'ensemble du Tibet ethnique en 1998.

1997 : Mort de Deng Xiaoping.

2000 : L'un des principaux hiérarques vivant encore au Tibet, le dix-septième Karmapa Ugyen Trinle Dorje (tib. U rgyan phrin las rdo rje), alors âgé de 14 ans, s'enfuit du Tibet vers l'Inde.

2001 : Début de la construction de la ligne de chemin de fer Golmud-Lhassa (1 $142 \mathrm{~km})$. Le dalaï-lama rencontre le président des États-Unis.

2002 : La RPC et le gouvernement en exil de Dharamsala reprennent des relations interrompues depuis 1993.

2003 : Deuxième session de pourparlers RPC/Dharamsala.

2004 : Troisième session de pourparlers RPC/Dharamsala. Le gouvernement chinois publie le «Livre blanc » sur « l'autonomie régionale ethnique au Tibet» dans lequel il exclut toute critique et toute négociation au sujet de l'autonomie existante et demande que le dalaï-lama renonce à ses « position en faveur de l'indépendance $»$.

$2005: 40^{\mathrm{e}}$ anniversaire de la RAT. Début de la rénovation et reconstruction de Lhassa avec des fonds gouvernementaux de Pékin. Quatrième session de pourparlers RPC/Dharamsala.

2006, 25 mai : Le Sénat américain décrète l'attribution de la médaille d'or du Congrès au dalaï-lama.

$\mathbf{1}^{\text {er }}$ juillet : Inauguration de la ligne de chemin de fer Golmud-Lhassa.

6 juillet : Réouverture de la route commerciale entre l'Inde et le Tibet par le col de Nathula (Sikkim).

13 septembre : La Chambre des représentants américaine vote l'attribution de la médaille du Congrès au dalaï-lama.

Plan quinquennal en RAT (2006-2010) de sédentarisation et de relocalisation des ruraux.

Cinquième session de pourparlers RPC/Dharamsala.

2007, 15 février : Le Parlement européen adopte une résolution sur le dialogue entre le gouvernement chinois et les envoyés du dalaï-lama dans le sens d'une approche plus ferme de l'Union européenne.

15 octobre : Ouverture du XVII ${ }^{e}$ congrès du Parti communiste chinois à Pékin. La RAT est fermée au tourisme pendant toute la durée du congrès.

17 octobre : Le Congrès américain décerne au dalaï-lama sa médaille d'or. Grande tension au Tibet. 
2008, mars-mai : Manifestations et émeutes d'une ampleur sans précédent depuis 1989 dans toutes les régions de culture tibétaine de la RPC : 125 manifestations, dont quatorze avec dégradations de biens. D'après le gouvernement tibétain en exil, il y aurait eu 203 morts. Le gouvernement chinois reconnaît un mort du côté tibétain et affirme que les émeutiers sont responsables de vingt et un décès. Des manifestations sporadiques se poursuivent, ainsi que les arrestations et séances intensives de rééducation politique.

8 mai : La flamme olympique est portée en haut de l'Everest.

12 mai : Un violent séisme frappe le Sichuan dont la région de population tibétaine de Ngawa.

21 juin : La flamme olympique passe par Lhassa.

\section{Bibliographie}

Anne-Marie Blondeau, Katia Buffetrille (éd.), Authenticating Tibet. Answers to China's 100 Questions, University of California Press, Berkeley, 2008, 463 p.

Anne Chayet, Art et archéologie du Tibet, Picard, Paris, 1994, 247 p.

Melvyn C. Goldstein, A History of Modern Tibet, 1913-1951, The Demise of the Lamaist State, Munshiram Manoharlal Publishers, New Delhi, [1989] 1993, 898 p.

Melvyn C. Goldstein, The Calm before the Storm, 1951-1955, University of California Press, Berkeley, 2007, 639 p.

Fabienne Jagou, Le 9e Panchen Lama (1883-1937) : enjeu des relations sino-tibétaines, École française d'Extrême-Orient, Paris, 2004, 431 p.

Matthew T. Kapstein, The Tibetans, Blackwell Publishing, Oxford, 2006, 360 p.

Françoise Pommaret, Tibet, une civilisation blessée, Paris, Gallimard, 2002, 159 p.

Tsering W. Shakya, The Dragon in the Land of Snows. A History of Modern Tibet Since 1947, Londres, Pimlico, 1999, 574 p.

David Snellgrove, Hugh Richardson, A Cultural History of Tibet, Shambala Publications, Boston et Londres, [1968] 1995, 309 p.

Rolf A. Stein, La civilisation tibétaine, L'Asiathèque-Le Sycomore, Paris, [1962] 1981, 312 p.

Heather Stoddard, Le mendiant de l'Amdo, Société d'ethnographie, Paris, 1985, 395 p. 